\title{
Paradigm Shift in the Treatment of Elderly Patients With Unresectable Stage IV Colorectal Cancer
}

\author{
Nam Kyu Kim \\ Department of Surgery, Yonsei University College of Medicine, Seoul, Korea
}

\section{See Article on Page 175-181}

Colorectal cancer (CRC) is one of the most common malignant tumors that lead to cancer-related death worldwide, and it has an increasing incidence rate. Approximately $20 \%$ to $30 \%$ of patients with CRC are found to have distant metastases at the time of diagnosis [1]. To date, with recent advances in systemic chemotherapy, nonoperative treatment has become the mainstay of treatment in patients with incurable metastatic CRC $[2,3]$. Given the progress in chemotherapy regimens and palliative techniques such as endoluminal stenting and given the high mortality of cancer-directed surgery, the prognostic impact of a primary tumor resection in patients with stage IV CRC has been under debate [46]. A previous analysis of Surveillance Epidemiology and End Results (SEER)-Medicare-linked data between 1991 and 1999 reported a $72 \%$ cancer-directed surgery rate in elderly patients (age $\geq 65$ ) with stage IV CRC [6]. However, more recent SEER-Medicare data from 2001 to 2007 demonstrated a statistically significant decrease in the rate of surgical resections of primary tumors from $64.6 \%$ in 2001 to $57.1 \%$ in 2007 [7]. Similarly, The Netherlands' Cancer Registry also found a significant decrease in primary tumor resection rates from $66 \%$ to $56 \%$ and an increase in systemic chemotherapy from $2.0 \%$ in $1989-1993$ to $40 \%$ in $2004-$ 2006 in elderly patients with stage IV CRC disease [8]. These treatment trends may indicate that practitioners are rapidly adopting the use of modern combination chemotherapy and that a paradigm shift in the treatment of stage IV CRC is beginning.

Current practice guidelines from the National Comprehensive

Correspondence to: Nam Kyu Kim, M.D.

Department of Surgery, Yonsei University College of Medicine, 50 Yonsei-ro, Seodaemun-gu, Seoul 120-749, Korea

Tel: +82-2-2228-2117, Fax: +82-2-313-8289

E-mail:namkyuk@yuhs.ac

(C) 2014 The Korean Society of Coloproctology

This is an open-access article distributed under the terms of the Creative Commons Attribution NonCommercial License (http://creativecommons.org/licenses/by-nc/3.0) which permits unrestricted noncommercial use, distribution, and reproduction in any medium, provided the original work is properly cited.
Cancer Network (NCCN) recommend a resection of the primary tumor only if patients are symptomatic or have metastatic sites amenable to a potentially-curative resection. Although the rationale for an up-front primary tumor resection is based on an attempt to resolve potential complications from the primary tumor, such as bowel obstruction, uncontrollable bleeding, or tumor perforation $[6,9]$, the critical role of surgery for patients with incurable stage IV CRC is still inconclusive, especially for patients with stage IVB CRC. Furthermore, proper prognostic clinical parameters, such as age, performance status, pathological grading, or localization of the primary tumor, should be considered before undertaking surgery in elderly patients with stage IV CRC. Although very limited evidence is available with respect to the survival benefit of a primary tumor resection in patients with stage IV CRC, recent studies demonstrated a $31 \%$ reduction in mortality with surgical resection of the primary tumor and an increase in survival in elderly patients with stage IV CRC $[10,11]$. Consistent with previous studies, our retrospective study showed that patients who underwent a primary tumor resection followed by chemotherapy had a significantly longer survival time than patients who received first-line chemotherapy (17.2 vs. 13.6 months, $\mathrm{P}=0.002$ ) [12]. On the basis of the current limited results, a primary tumor resection is potentially associated with survival benefits in elderly patients with stage IV CRC. However, primary-tumor-related complications are a major concern. The rates of complication in patients with an unresected primary tumor have been reported to range from $11.0 \%$ to $30 \%[2,13,14]$.

This study analyzed retrospectively the survival outcomes and the prognostic factors in elderly patients with stage IVB CRC. The current study showed that the patients in the resection group had significantly longer median survival times compared to the patients in the nonresection group (12.43 months vs. 3.58 months, $\mathrm{P}$ $<0.001)$. In addition, male gender, higher level of carcinoembryonic antigen, higher aspartate aminotransferase level, and nonresection of the primary tumor were independent poor prognostic factors on the multivariate analysis. However, the relatively short follow-up time and the small sample size are major drawbacks to interpreting the survival benefits and the prognostic factors 
clearly [15]. A large-scale, randomized study is needed to clearly establish the true benefits primary tumor resection for stage IVB $\mathrm{CRC}$. In conclusion, the appropriate role of a surgical resection for treating stage IVB CRC, including its prognostic impact on tumor-related symptom control, its survival benefits and its impact on quality of life, remains to be fully evaluated.

\section{REFERENCES}

1. Ferrand F, Malka D, Bourredjem A, Allonier C, Bouche O, Louafi $S$, et al. Impact of primary tumour resection on survival of patients with colorectal cancer and synchronous metastases treated by chemotherapy: results from the multicenter, randomised trial Fédération Francophone de Cancérologie Digestive 9601. Eur J Cancer 2013;49:90-7.

2. Poultsides GA, Servais EL, Saltz LB, Patil S, Kemeny NE, Guillem JG, et al. Outcome of primary tumor in patients with synchronous stage IV colorectal cancer receiving combination chemotherapy without surgery as initial treatment. J Clin Oncol 2009; 27:3379-84.

3. Scheer MG, Sloots CE, van der Wilt GJ, Ruers TJ. Management of patients with asymptomatic colorectal cancer and synchronous irresectable metastases. Ann Oncol 2008;19:1829-35.

4. Basili G, Lorenzetti L, Biondi G, Preziuso E, Angrisano C, Carnesecchi $\mathrm{P}$, et al. Colorectal cancer in the elderly. Is there a role for safe and curative surgery? ANZ J Surg 2008;78:466-70.

5. Konyalian VR, Rosing DK, Haukoos JS, Dixon MR, Sinow R, Bhaheetharan $S$, et al. The role of primary tumour resection in patients with stage IV colorectal cancer. Colorectal Dis 2007;9: 430-7.

6. Temple LK, Hsieh L, Wong WD, Saltz L, Schrag D. Use of surgery among elderly patients with stage IV colorectal cancer. J Clin Oncol 2004;22:3475-84.

7. Vargas GM, Sheffield KM, Parmar AD, Han Y, Gajjar A, Brown $\mathrm{KM}$, et al. Trends in treatment and survival in older patients pre- senting with stage IV colorectal cancer. J Gastrointest Surg 2014; 18:369-77.

8. van Steenbergen LN, Elferink MA, Krijnen P, Lemmens VE, Siesling S, Rutten HJ, et al. Improved survival of colon cancer due to improved treatment and detection: a nationwide populationbased study in The Netherlands 1989-2006. Ann Oncol 2010;21: 2206-12.

9. Damjanov N, Weiss J, Haller DG. Resection of the primary colorectal cancer is not necessary in nonobstructed patients with metastatic disease. Oncologist 2009;14:963-9.

10. Ahmed S, Leis A, Fields A, Chandra-Kanthan S, Haider K, Alvi R, et al. Survival impact of surgical resection of primary tumor in patients with stage IV colorectal cancer: results from a large population-based cohort study. Cancer 2014;120:683-91.

11. Ahmed S, Shahid RK, Leis A, Haider K, Kanthan S, Reeder B, et al. Should noncurative resection of the primary tumour be performed in patients with stage iv colorectal cancer? A systematic review and meta-analysis. Curr Oncol 2013;20:e420-41.

12. Kim MS, Chung M, Ahn JB, Kim CW, Cho MS, Shin SJ, et al. Clinical significance of primary tumor resection in colorectal cancer patients with synchronous unresectable metastasis. J Surg Oncol 2014;110:214-21.

13. Bajwa A, Blunt N, Vyas S, Suliman I, Bridgewater J, Hochhauser D, et al. Primary tumour resection and survival in the palliative management of metastatic colorectal cancer. Eur J Surg Oncol 2009; 35:164-7.

14. Galizia G, Lieto E, Orditura M, Castellano P, Imperatore V, Pinto $\mathrm{M}$, et al. First-line chemotherapy vs bowel tumor resection plus chemotherapy for patients with unresectable synchronous colorectal hepatic metastases. Arch Surg 2008;143:352-8.

15. Ahn HJ, Oh HS, Ahn YC, Lee SJ, Kim HJ, Kim MH, et al. Prognostic implications of primary tumor resection in stage IVB colorectal cancer in elderly patients. Ann Coloproctol 2014:30; 175-81. 